\title{
Neuronal and Inducible Nitric Oxide Synthase Expression in Different Regions of Rat Brain in Kainic Acid Induced Epilepsy by Immunohistochemical Analysis
}

\author{
Swamy $\mathrm{M}^{*}$, Intan NMZ, Sirajudeen KNS, Zulkarnain M and Chandran G \\ Department of Chemical Pathology, School of Medical Sciences, Health campus, Universiti Sains Malaysia, 16150 \\ Kubang Kerian, Kelantan, Malaysia; E-mail:mswamy@kb.usm.my
}

Kainic acid (KA) is a natural product derived from the seaweed, Digenea simplex. It is frequently used as a tool to experimentally mimic human temporal lobe epilepsy. Nitric oxide (NO) is known to be involved in the pathophysiology of many epilepsy models. The aim of this study was to determine the immunoreactivity of neuronal and inducible nitric oxide synthetase (nNOS \& iNOS) in different regions of brain in rats subjected to KA induced epilepsy. Male Sprague-Dawley rats were used in this study. The acute group rats were sacrificed after 2 hours and the chronic group rats were sacrificed after 5 days of single injection of KA $(15 \mathrm{mg} / \mathrm{kg}$ body weight) and the cerebral cortex $(\mathrm{CC})$, cerebellum(CB) and brain stem (BS) slices were constructed using formalin-fixed, paraffin embedded tissues. Those sections were immunohistostained with commercial antibodies for the above enzymes. Control group rats received normal saline and processed in a similar manner. Images were captured. Results were analyzed by one-way ANOVA and reported as mean \pm standard error from 6 animals and p<0.05 considered statistically significant. In acute group nNOS immunoreactivity was increased in CC and BS and iNOS immunoreactivity was increased in $\mathrm{CC}$ and $\mathrm{CB}$ compared to control group. In chronic group both nNOS and iNOS immunoreactivity was increased compared to control in all the brain regions studied. The study clearly demonstrated the up regulation of nNOS and iNOS in KA treated rats and may support the increased production of NO in epilepsy which may be responsible for neurodegeneration. 